\title{
INITIAL PERFORMANCE OF THE WIRE IMAGING SYNCHROTRON RADIATION DETECTOR*
}

\author{
C. Von Zanthicr, J,-J. Gomez Cadenas, J. Kent, M. King, and S. Watson \\ liniversity of California at Santa Crus, Santa Cas, California 95054 \\ D. D. Briggs, F. Rouse, and J. Tinsman \\ Stanjord Lincar Accelerator Center, Stanford Cniversty, Stanford, CA 94909 \\ SLAC-PUB- -5112 \\ DE90 00645 ?
}

Abstract

This paper describes the initial performance of a novel detector that measures the positions of intense synchrotron-radiation beams with high precision. Two detectors of this kind are used for the precision energy spectrometers of the Stanford Linear Collider (SLC). The detectors accurately determine the distance between pairs of intense synchrotron bearrs of typically $1 \mathrm{MeV}$ photons, which are emitted by the primary electron and positron beams of the SLC. The delectors intercept the synchrotron beams with array's of fine wires. The ejection of Compton-recoil electrons leaves positive charges on the wires, enabling a determination of bean positions.

\section{INTHODUCTION}

The Wire Inaging Synchrotron Radiacion Detector (WISRD) [1] is a novel detector for the position measurement of intense high-energy synchrotron radiation beams. Two WISRDs are installed for the two precision energy spectrometers [2] of the SLC. The spectrometers are used to determine center-of-mass energies of the colliding electron and position beams.

Figure 1 shows schematically the energy spectrometer for the electron beam. Two horizontally bending dipole magnets, one upstream and one downstream the vertically bending spectrometer magnet, generate two horizontal suaths of intense tynchrotron radiation. The photons of the synchrotron radiation stripes have an energy typically of $1 \mathrm{MeV}$ (the critical energy $E_{\mathrm{e}}$ of the photan energy spectrum is $E_{e} \approx 3 \mathrm{MeV}$ ). The full width of the stripe at the detector is of the order of $1000 \mu \mathrm{m}$. Given the strength of the analyzing magnet and its distance upstream from the detectors, the vertical separation between the two synchrotron stripes determines the SLC beam energy. The vertical separation is about $0.27 \mathrm{~m}$. The goal for the WISRD is to measure this distance within a few parts per ten-thousands. This measurement will be independent Irom the measurement performed by the Phosphor Screen Monitor (PSM) [3], which has been used until now for the determination of the SLC center-of-mass energies.

\footnotetext{
* Work supported by Department of Energy contracts DEAC03-76SF00515, DE-AC03-76SF00098, and DE-AM03$76 S F 00010$.
}

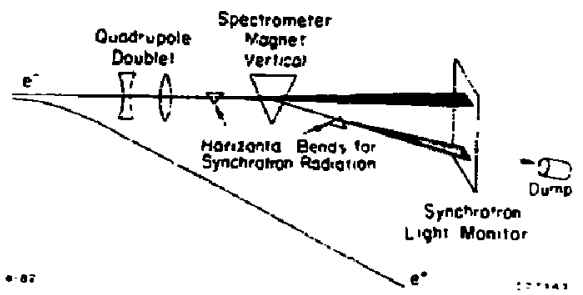

Fig. 1: Conceptual design of the extraction line spectrometer.

This paper briefly reviews the WISRD system and reports on its initial perlormance. More details about the detector design and read-out electronies can be found elsewhere $[1,4,5]$.

\section{DEscaiption OF THE WISRD SYSTEM}

The WISRD for the SLC electron spectrometer is shown in Fig. 2. The detector for the positron spectrometer is virtually identical and not explicitly discussed here. At the heart of the detector are two arrays of ver $y$ fine copper wires, which serve as targets for the two collimated horizontal Bynchrotron radiation stripes. Both arrays consist of 96 horizontal wires. The wrires are $75 \mu \mathrm{nin}$ in diameter and have a $100 \mu \mathrm{m}$ center-to-senter spacing.

The wires intercepting the synchrotron radiation photons eject electrons due to predomizately Compton seattering. The synchrotron eadiation is sufficiently intense to result in positive charges on the wires that can be measured directly. The observed charge as a function of the wire aumber allows the reconstruction of the profiles and hence the centroids of the two synchrotron stripes. Since the wire positions relative to each other are known to bigh precision, the vertical distanse between the two stripes can be determined.

Numerical calculations and Monte Carlo simulations predict a total charge of $180 \mathrm{fC}$ per stripe distributed over all wires for an electron bunch of $1-10^{10}$ electrons and for

Contributed to the JEEE Nuclear Science Symposium, 


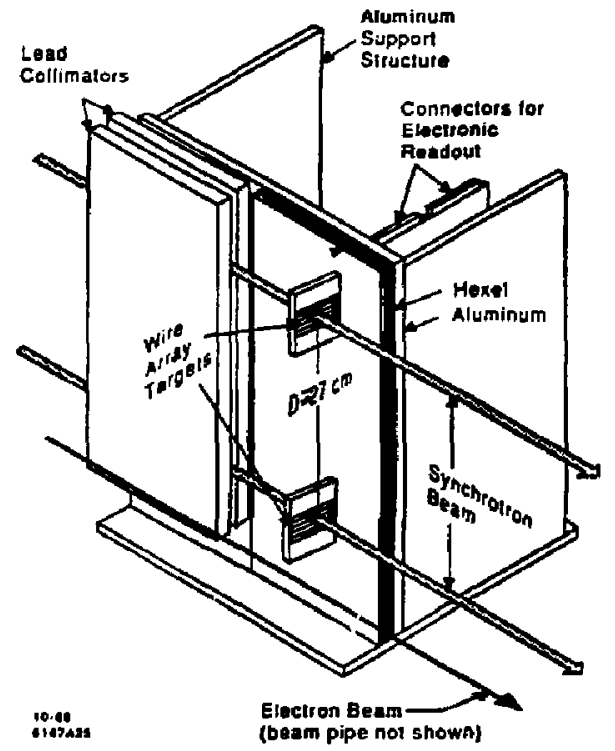

Fig. 2: An isometric view of the wire imaging synchrotron radiation detector (WISRD).

Q stripe length collimated to $1 \mathrm{~cm}$. This signal is detected with the data acquisition system shoren in Fig. 3 . Each detector wire is connected to one channel of a LeCroy HQV820 charge sensilive amplifier followed by a pulse-shaping circuil. Twenty-four shaped pulses are sampled twice by a parallel-in. serial-out analog memory circuit iCDC : $i^{2}$, The timing of the samples is controlled by sofiware The serial output of the analog memory circuit is digitized by a 12-bit ADC, then encoded and transmilted through a 300-m-long cable to a CAMAC data acquisit on systen: A VAX $\mathbf{8 6 0 0}$ reads the CAMAC erate and writes the dat a to tape.

The proximity of the WISRD is a barsh envitonment for electronics. To suppress electrornagnetic interference. the electronics show $n$ in Fig. 3 is housed in a RF shrelding box. The RF box is located abous $4 \mathrm{~m}$ axa! from the detector to avoid radiation damage to the electronies from the synchrotron radiation beam halo.

\section{INITIAL HISRD DATA}

Initial data have verified the basic components of the WISRD system and provided pointers for further inprovements.

The WISRD electronies has been designed to allow extensive testing and calibration of the system with test pulses [4]. Out of the nearly 400 channels of electron. ics, $A$ were found to be dead. A major challenge in the fabrication of the detector target arrays was to avoid accidental electrical contacts between pairs of detector wires: only four detector wire pairs were found to be shorted. The cross talk between channels due to coupling in the detector and the electronics is $5 \%$ on average.

The calibration system permits reconstruction of the characteristic bipolar pulse at the input of the sample and hold circuit. Figure 4 show's such a measurement for

DATA ACQUISITION MODULE BLOCK DIAGRAM

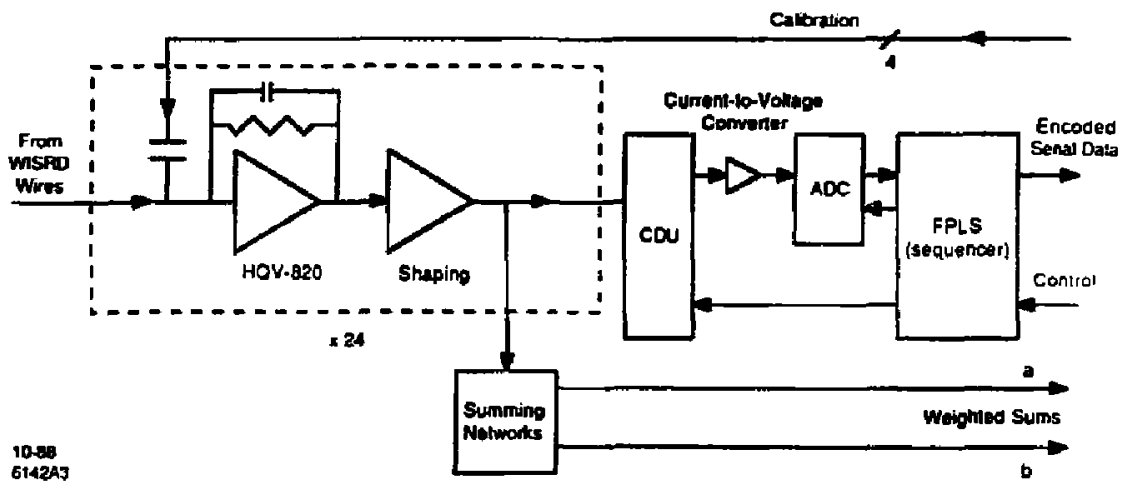

Fig. 3: The Data Acquisition (DAQ) Module receives signals from 24 wires in the WISRD wire array. amplifies and shapes the signals, and stores two samples of each shaped output in a parallel-in, serial-out analog memory (CDC') on every beam crossing. After data acquisition, the analog samples are digitized and encoded for transmission The DAQ Mlodule also forms two weighted sums of the chage distribution on its wires. 


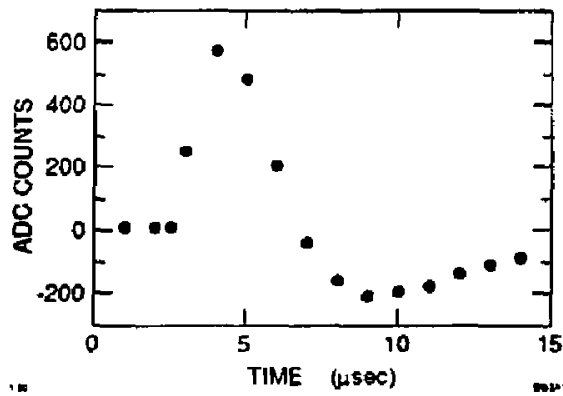

Fig A: A calibration pulse after shaping and before sampling with the $C D L^{\circ}$ chip. 'This shape has been reconstructed by taking the difference of the two CDI samples. where ote sampling occurs shortly before the calibration pulse onset and the other sampling is scarned in I usec steps across the pulse.
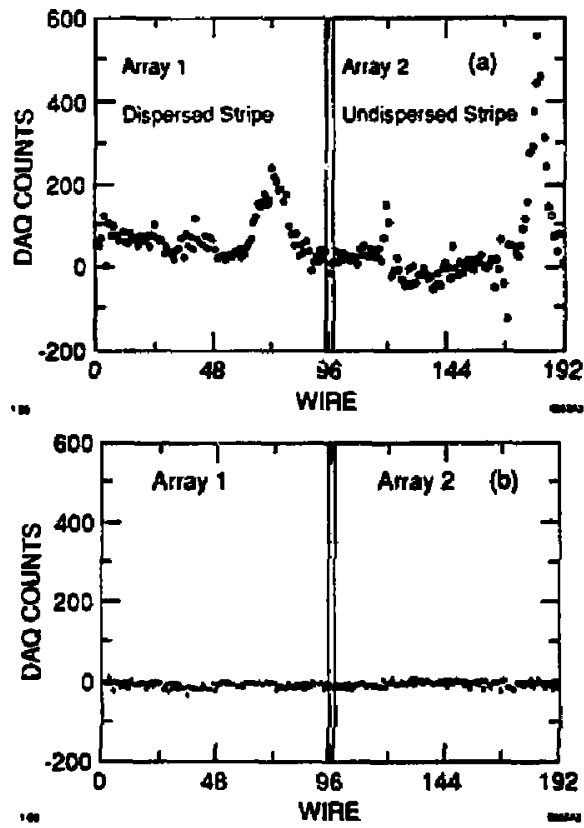

Fig. 5: (a) Typical signal distribution from the two synchrotron radiation stripes hitting the two wire as rays. The right peak is from the stripe emitted by the undispersed electron beam; the left peak, from the stripe emitted by the dispersed electron beam (b) Same plot as (a); however, the signal for each wire has been sampled immediately before beam by-passing. As in (a), no background is seen.

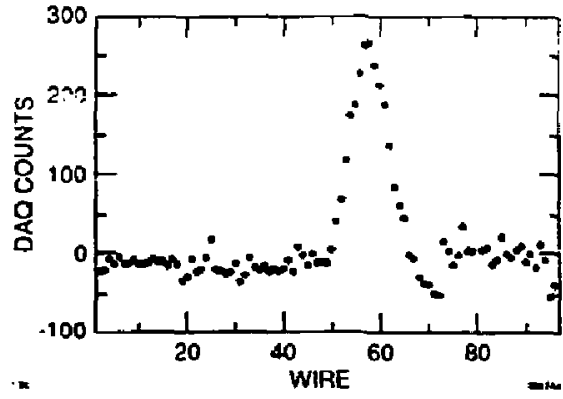

Fig 0 A synchrotron stripe (integrated over 10 pulses) as seen in an earlier electronics and dara acquisition ronfiguration. This plot show's that beam-correlated background as in Fig. $5(a)$ can be widely suppressed.

a tipical channel. Such data are useful for luning the sampling times for optimization of signal amplitude and background suppression. In normal data analysis. the signal amplitude for a wire is computed by subtracting the two recorded samples of the analog pulse shape. Sominally: the first sampling time is on the posilive peak and the second sampling time is on the negative peak

The data presented in Fig. 5 (a) show signals corresponding to one SLC pulse containing roughly $10^{\circ} \mathrm{C}$ elec. trons. The signals are corrected for gain and pedestal variations. Wire numbers from 0 to 95 refer to the lower wire assay in Fig. 2. and the remaining wire numbers correspond to the upper array. The peaks centered around wires 75 and 180 are due to the synchrotron beans. In addition to the desired signals, beam-correlated backgrounds are present, as can be varified by shifting the sampling times to times just befote the arrival of the beams; see Fig. 5(b).

Work is in progress to reduce the beam-correlated backgtound. Initial data provide guidance for further improvernents of the systern: sample time adjustments: pulse shape optimization; and electromagnetic shielding improvements. The background has been observed to vary significantly with these parameters; for example see Fig. 6. which shows data from on preliminary test configuration. W'ith improvements in both hardware and softuare, it is anticipated that beam-rorrelated backgrounds will be effectively suppressed.

The prominent synchrotron-beam signals seen in Fig. 5 are lypical of data that are now routinely collected by the WISRD system. They show the fealures that were anticipated in the detector design. The right peak seen in Fig. $5(a)$ is produced by the synctrotron stripe emitted upstream of the spectromete: magnet by the SLC electron beam. Monte Carlo simulations imply that this width is dominated by the $1 / \gamma$ production angle characteristic of synchrotron radiation. The left peak is produced by the 
synchrotron stripe emilled by the SLC electron beam after being dispersed by the spectrometer magnet: hence it is vider. The expectation that the areas under the two peaks be equal is also consistent with the data

\section{Discl's5ioN OF ERRORS}

A design goal for the WISRD is to determune the positions of the centroids to $25 \mu \mathrm{m}$. This correspends to a contritution in the SLC beam energy esror of about 4 MrV Costributions to the measurement error come from uncertainties in the wire geometry, electronic noise, calibration constants uncertainties, and electromagnetic backgrounds:

- Data bases containing redundant measurements of wire positions are available for the determination of the wire geonet ry. The vertical distance between any Iwo ures in the detector can be calculated [1] to $10 \mu \mathrm{m}$.

- Electronic noise inherent to the electronic system contributes an uncertainty to the centroid measurement. This centroid uncertainty contribution has been estimated [4] 10 be $9 \mu \mathrm{m}$ for beam currents of $10^{10}$ electrons per pulse. Sote that the effecl of random noise reduces in significance for analysis involving data from more than one SLC beam pulse.

- Calibration data are used in the calculation of gais and pedestal corrections of the data Calibration uncertainties contribute [4] Iypically $10 \mu \mathrm{m}$ to the centroid error.

The conbined effect of the above three sources of error is about $17 \mu \mathrm{m}$. We are confident that the beamcorrelated barkground can be sufficiently suppressed by hardware and software to meet the design goal of $25 \mu \mathrm{m}$.

\section{Concllision}

The WISRD is a novel detector that monitors with high precision the positions of intense high-nergy synchrotron tadiation beams. It is going to be a key element of the precision spectrometers that records the center-ofmass energies of the SLC.
The WISRD detector and its electronics are in placm and fully operational. The expected signal duf ic thr synchroiron bearns has been confirmed This validates the design of detector and its electronirs Prithmuats? measurements indicate that it is possible to deternuthe thr synchrotron st ripe positions to bettet than $2 \% \mu \mathrm{m}$

\section{ACKYOWLEDGMENTS}

11. Petree and D Wilkinson are thanked fres lhelf thorcugh work on the electronics for both delector sy:tems A Kaluzniackl is acknowledged for his contritutions to the analssis of the data

\section{References}

1 J Kent el al. Design of a Wire-Imaging Synchrotron Radiation Detertor. IEEE 19R9 Suclear Science Symposium. San Francisco. CA. January 15-19. 1990. SLAC-P['B-51]0.

2. J Kent ef al. Precision Measurement of tife SLC Beam Beam Energy, SLAC-PLB-4922. LBL-2697\%. IEEE Particle Actelerator Conference. Chicago. $1 \mathrm{~L}$. March 20-23, 1989

3 M. Levi et al. Precision Synchrolron Radiation Drtectors. SLAC-P['B-4921. LBL-26976. IEEE Part1cle Accelerator Conference. Chicago. 1L. March 2023. 1989 .

4. F. Rouse ef al. The Electronics and Data Acquisition System for the VIre.Imaging Synchrot ron Radiation Detector at the SLC. IEEE 1989 Nuclear Science Symposium. San Francisco. CA, January 15-19. 1990. SLAC-Pl'B-5II1

5. D. D. Briggs el al., A CDl-Based Data Arquistuon System for for the Energy: Specirometer at the Sianford Linear Collider. SLAC-PLB-473i. LBL-26243. IEEE 1989 Xuclear Science Sy'mposium. Orlaudo. Florida. November 9-11, 1988.

6. G. Haller of al. Performance Report for Stanford/SLAC Multi-Channel Sample-and-Hold Device. IEEE Trans. Nucl Sci NS-33. 221 (1986)

\section{DISCLAIMER}

This report was prepared as an accounl bif uork sponsored by an agency of the United States Government. Neither the United States Government nor any agency thereof. nor any of their emplayees, makes any warranty, express or implied, or assumes any legal liabiluty ur responsibility for the oceuracy, completeness, ur usefuiness of any information, apparatus, product, of prucess disclosed, or represents thal its use wauld not infringe privalely uwned rights. Reference herein to any specific commercial product, process, or service by trutle name. trademark. manufucturer, or otherwise does not necessarily constitule or imply its endorsement, recom. mendation, or favoring by the United States Government or any agency thereof The views and opinouns of authors expressed herein do not necessarily state of reflect those ur the United Stetes Government or any igency thereor. 